\title{
Low-mass companions to Hyades stars ${ }^{\star}$
}

\author{
E. W. Guenther ${ }^{1}$, D. B. Paulson ${ }^{2}$, W. D. Cochran ${ }^{3}$, J. Patience ${ }^{4}$, A. P. Hatzes ${ }^{1}$, and B. Macintosh ${ }^{5}$
}

\author{
1 Thüringer Landessternwarte Tautenburg, Sternwarte 5, 07778 Tautenburg, Germany \\ e-mail: guenther@tls-tautenburg.de \\ 2 Planetary Systems Branch, Code 693, NASA Goddard Space Flight Center, Greenbelt, MD 20771, USA \\ 3 McDonald Observatory, University of Texas at Austin, Austin, TX 78712, USA \\ 4 Division of Physics, Mathematics, and Astronomy, California Institute of Technology, Pasadena, CA, USA \\ 5 Institute of Geophysics and Planetary Physics, Lawrence Livermore National Laboratory, 7000 East Avenue L-413, Livermore, \\ CA 94551, USA
}

Received 9 February 2005 / Accepted 20 July 2005

\section{ABSTRACT}

It is now well established that a large fraction of the low-mass stars are binaries or higher order multiples. Similarly a sizable fraction have giant planets. In contrast to these, the situation for brown dwarf companions is complicated: While close systems seem to be extremely rare, wide systems are possibly more common. In this paper, we present new results on a survey for low-mass companions in the Hyades. After measuring precisely the radial velocity of 98 Hyades dwarf stars for 5 years, we have selected all stars that show low-amplitude long-period trends. With AO-observations of these 14 stars we found companion candidates around nine of them, where one star has two companions. The two companions of HIP 16548 have masses between 0.07 to $0.08 M_{\odot}$, and are thus either brown dwarfs or very low mass stars. In the case of HAN 172 we found a companion with a mass between 0.08 to $0.10 M_{\odot}$, which is again between a star and a brown dwarf. The other seven stars all have stellar companions. In two additional cases, the $R V$-variations are presumably caused by stellar activity, and in another case the companion could be a short-period binary. The images of the remaining two stars are slightly elongated, which might imply that even these are binaries. Because at least 12 of the 14 stars showing low-amplitude $R V$ trends turn out to have companions with a mass $\geq 70 M_{\mathrm{Jupiter}}$, or are just active, we finally estimate the number of companions with masses between $10 M_{\text {Jupiter }}$ and $70 M_{\text {Jupiter }}$ within 8 AU of the host stars in the Hyades as $\leq 2 \%$.

Key words. stars: low-mass, brown dwarfs - stars: binaries: spectroscopic - stars: binaries: visual - stars: planetary systems

\section{Introduction}

It is now well established that most of the solar-like stars are binaries (Abt \& Levy 1976; Duquennoy \& Mayor 1991). A detailed analysis shows that $13 \pm 3 \%$ of the G stars, and $8.1 \%$ of the M stars are binaries with separation of $3 \mathrm{AU}$, or less (Mazeh et al. 1992; Fischer \& Marcy 1992). The frequency of planets with a mass of $m \sin i \geq 0.3 M_{\text {Jupiter }}$ orbiting at distances $\leq 5 \mathrm{AU}$ is about $9 \%$ (Lineweaver \& Grether 2003). It is quite surprising that brown dwarfs are very rare as close companions to normal stars. The lack of brown dwarfs as companions

* Partly based on observations obtained at the European Southern Observatory at Cerro Paranal, Chile in program 68.C-0063(A), 72.C0288(A) and 072.C-0110(B), and partly based on observations obtained at the W.M. Keck Observatory through telescope time allocated to the National Aeronautics and Space Administration through the agency's scientific partnership with the California Institute of Technology and the University of California. The Keck observatory was made possible by the generous financial support of the W.M. Keck Foundation. Also partly based on observations obtained at the Magellan Telescope. is thus often referred as the brown dwarf desert. Marcy et al. (2003) estimate from their radial velocity $(R V)$ survey that the frequency of brown dwarfs with $3 \mathrm{AU}$ of the host stars is only $0.5 \pm 0.2 \%$, and thus much smaller than the frequency planets, or the frequency binaries. Studies by Zucker \& Mazeh (2001) show that the frequency of close companions drops off very sharply for masses higher than $10 M_{\text {Jupiter }}$, although they suspect there is still a higher mass tail that extends up to probably $20 M_{\text {Jupiter }}$. From the currently known 161 "planets" 8 have an $m \sin i$ between 10 and $18 M_{\text {Jupiter }}{ }^{1}$. There are only very few brown dwarfs known that orbit normal stars at distances $\leq 3$ AU (Zucker \& Mazeh 2000; Udry et al. 2002; Endl et al. 2004).

In the case of wide pairs consisting of a brown dwarf and a star, the situation is more complicated. These wide companions (e.g. $d \geq 50 \mathrm{AU}$ ) are usually detected by means of direct imaging. Direct imaging campaigns probably have turned up a number of brown dwarfs orbiting normal stars

\footnotetext{
1 As compiled from J. Schneiders "Extrasolar Planets Encyclopaedia", http://www.obspm.fr/encycl/catalog.html").
} 
(Gliese 229B: Nakajima et al. 1995; TWA-5 B: Lowrance et al. 1999; Neuhäuser et al. 2000; HR 7329 B: Lowrance et al. 2000; Guenther et al. 2001; Gliese 417B and Gliese 584C: Kirkpatrick et al. 2001; HR 7672: Liu et al. 2002; HD 130948 (two brown dwarfs): Goto et al. 2002, Potter et al. 2002; LHS 2397aB: Freed et al. 2003, Masciadri et al. 2003; GSC 08047-00232: Neuhäuser \& Guenther 2004; $\epsilon$ Indi B: McCaughrean et al. 2004; G124-62 Seifahrt et al. 2005). Additionally to these detections there are three objects that are either low-mass brown dwarfs, or even giant planets (Neuhäuser et al. 2004; Chauvin et al. 2005a,b). The result of all search programs for objects in TWA-Hydra, Tucanae, Horologium and the $\beta$ Pic region is that the frequency of of brown dwarfs at distances larger than $50 \mathrm{AU}$ is $6 \pm 4 \%$ (Neuhäuser et al. 2003). This result implies that the frequency of wide binaries consisting of a brown dwarf and a star is much higher than that of close binaries. One possible explanation for these results may simply be the difference of the methods used for detecting short and long period systems. In the case of the long-period systems, the masses of these objects have been determined from absolute brightnesses by evolutionary tracks. If for some reason the evolutionary tracks are off by a certain factor, a number of these brown dwarfs would be stars. There are in fact notable differences between evolutionary tracks published by different authors, especially for objects of lower mass, and young age (Wuchterl \& Tscharnuter 2003; Stern 1994; Burrows et al. 1997; Malkov et al. 1998; Chabrier et al. 2000). A recent determination of the dynamical mass of $\mathrm{AB}$ Dor $\mathrm{C}$ indicates that the true mass is a factor of two higher than the mass derived from evolutionary tracks, converting this brown dwarf into a star (Close et al. 2005). On the other hand, in the case of binary brown dwarf GJ 569 Bab the true mass and the mass derived from evolutionary tracks agree reasonably well (Zapatero Osorio et al. 2004). Possible explanations for the discrepancy could be that $\mathrm{AB}$ Dor $\mathrm{C}$ is a binary, or that AB Dor has an age of only $50 \mathrm{Myr}$, whereas GJ 569 Bab one between 120 and 1000 Myr. A study of old, isolated G, K, and $\mathrm{M}$ stars indicate that there is also a brown dwarf desert for long period systems, as McCarthy \& Zuckerman (2004) derive a frequency of $1 \pm 1 \%$ for brown dwarfs orbiting stars between 75 and $300 \mathrm{AU}$.

We can thus summarize that there is a general agreement that the frequency of brown dwarfs orbiting stars at distances of $\leq 3 \mathrm{AU}$ is about $\leq 0.5 \%$. For brown dwarfs at larger distances, there are two somewhat contradicting results: one is that the frequency is $1 \pm 1 \%$, the other that it is $6 \pm 4 \%$.

The aim of this work is to shed more light on the brown dwarf desert problem by a novel approach: Brown dwarfs out to a distance of about $8 \mathrm{AU}$ can be detected by means of radial velocity measurements, because these would show up as trends in the radial velocity data, and can thus be detected in this way. The only problem is that a stellar companion at a larger distance will also induce a trend of the RV-data. The trick is that wide binary stars can easily be detected by means of adaptive optics (AO) imaging. Objects that show low-amplitude trends in the RV-data, and are not binary stars are thus brown dwarf candidates, or even giant planets. In other words, if all stars that show low-amplitude trends in the RV-data turn out to be binary stars, we can conclude that it is highly unlikely that any of the stars monitored has a brown dwarf companion orbiting the primary within $8 \mathrm{AU}$. The advantage of this method is that the selection of the objects is based on RV-measurements, and thus all brown dwarfs within $8 \mathrm{AU}$ of the host stars are detected, unless the orbit is very unfavorable.

For this project we select a cluster of stars: the Hyades. This has three advantages. First of all, a cluster is a homogeneous sample, as all stars have the same age, metallicity, and distance. Secondly, the Hyades are sufficiently young (625 \pm 50 Myrs, Perryman 1998; or 650 Myrs Lebreton et al. 2001) and close enough $(46.34 \pm 0.27 \mathrm{pc}$, Perryman 1998$)$ so that not only stellar companions can be detected but also brown dwarfs. The third reason is that this sample was neither studied by Neuhäuser et al. (2003), nor by McCarthy \& Zuckerman (2004) and thus serves as a independent investigation that can be compared with these results. Additionally, the Hyades are metal-rich $([\mathrm{Fe}] /[\mathrm{H}]=+0.17 \pm 0.06$ : Boesgaard \& Budge 1988; $[\mathrm{Fe}] /[\mathrm{H}]=$ $+0.12 \pm 0.03$ : Cayrel et al 1985; $[\mathrm{Fe}] /[\mathrm{H}]=+0.13 \pm 0.01$ : Paulson et al. 2003). Whether the frequency of brown dwarf companions is related to the abundance or not is not known but it is known that the frequency of planets is higher for metal rich stars (Santos et al. 2004). Also in this respect it is interesting to compare the frequency of brown dwarf companions of Hyades stars with that of normal stars.

\section{Observations}

\subsection{Radial velocity: the input sample}

The RV measurements were carried out with the HIRES spectrograph on the 10-m-Keck telescope over a period of about 5 years (see Cochran et al. 2002; Paulson et al. 2002 and Paulson et al. 2004 for details). After measuring the RVs of 98 Hyades dwarf stars for 5 years, we identified 13 stars that show long-period, linear trends. The objects selected have trends with a velocity gradient $(\Delta R V)$ between 5 and $100 \mathrm{~m} / \mathrm{s} \mathrm{yr}^{-1}$. At the elongation, the gradient for a Hyades-star in a circular orbit is $\Delta R V=$ $6.6\left(m_{\text {comp }} /\left[80 m_{\text {Jupiter }}\right]\right)(\mathrm{sep})^{-2}\left[\mathrm{~m} \mathrm{~s}^{-1} \mathrm{yr}^{-1}\right]$ (with $m_{\text {comp }}$ the mass of the companion, and sep the separation in arcsec). An object showing a trend of this order thus is most likely either a wide system consisting of two stars, or a system consisting of a star and a substellar-mass companion. By imaging these objects with an AO-system, we can distinguish between stellar- and substellar- mass companions. Combining RV-data with AO-imaging of relatively young objects also is a good way of finding suitable targets for attempts to directly image planets with future instruments like CHEOPS (Claudi et al. 2004). Table 1 gives a short overview of the objects chosen.

\subsection{Direct imaging: NACO}

Most of the direct images were carried with the NAOSCONICA-system (Nasmyth Adaptive Optics System - HighResolution Near IR Camera) at the VLT-UT 4 telescope Yepun of the European Southern Observatory at Cerro Paranal, Chile in program 72.C-0288. We used the S27-camera, which gives 
Table 1. The stars

\begin{tabular}{lllll}
\hline \hline & Spec & $m_{V}$ & RA (2000.0) & Dec (2000.0) \\
\hline HD 28099 & G2V & 8.1 & 042640.1 & +164449 \\
HD 30505 & & 9.0 & 044903.5 & +183828 \\
HIP 16908 & K2V & 9.4 & 033735.0 & +212035 \\
HD 286589 & K5V & 10.8 & 041451.9 & +130318 \\
LP 415-176 & K2V & 11.6 & 043144.5 & +153746 \\
HIP 16548 & M0V & 12.0 & 033305.3 & +045729 \\
V1102 Tau & M1V & 12.2 & 042828.7 & +174145 \\
HAN 513 & M0.5V & 12.3 & 043143.3 & +150229 \\
HAN 172 & M1V & 12.5 & 041747 & +133942 \\
HIP 15720 & K7V & 8.5 & 032228.1 & +270922 \\
BD+08 642 & K5V & 10.1 & 040949.4 & +091820 \\
BD+16630 & & & 043600 & +163230 \\
J 332 & K5V & 11.5 & 044735 & +145324 \\
LP415-378 & M0V & 12.4 & 042912 & +151612 \\
\hline
\end{tabular}

a field-of-view of $28 \times 28$ arcsec. An image scale of $27.07 \pm$ 0.02 mas/pixel was recently derived by Neuhäuer et al. (2004) using Hipparcos stars. The new value of the image-tilt is $0.14 \pm 0.10^{\circ}$ (towards the east). Our original plan was to observe all objects with and without a semi-transparent coronographic mask but not all observing blocks could be carried out.

The semi-transparent mask has a projected diameter of 0.7 arcsec, and dims the light of the central star by about 10 magnitudes. This mask is thus very useful for detecting faint objects at separations larger than 0.35 arcsec, and bright objects closer to the star. It also allows us to measure the position angles and separations if a companion is found. According to the evolutionary tracks calculated by Burrows et al. (1997) objects of $20 M_{\text {Jupiter }}$ have nearly the same brightness in the $J$, and in the $K$-band. Because with NACO a similar limiting magnitude is reached easier in in the $J$-band than in the $K$-band, we used $J$-band filter with a central wavelength $1.265 \mu \mathrm{m}$ for these observations. The exposure-time was set in order to reach a $3 \sigma$ limiting magnitude between $J=22.5 \mathrm{mag}$ and $J=23 \mathrm{mag}$. According to Masciadri et al. (2005) NACO allows to detect an object 12 mag fainter than the primary at the $5 \sigma$-level under average observing conditions. The limit thus is 20 to $22 \mathrm{mag}$ at a separation of one arcsec, which corresponds to objects with a mass of 10 to $20 M_{\text {Jupiter }}$ (using Burrows et al. 1997). We thus can not only detect companions stars but also the brown dwarfs themselves at distances larger than about $16 \mathrm{AU}$. Table 2 gives an overview of the data taken, where "coro" or "direct" indicates whether the semi-transparent coronographic mask was used, or not used. "SR" is a conservative estimate of the Strehl ratio. The shortest possible exposure time (0.3447 s) was used for "direct" observations in order to minimize, or avoid the saturation of the primary stars. Because of the corresponding increase of the read-out noise, the on-source observation time had to increased in order to reach about the same limiting-magnitude, as in the coronographic images. The data was taken in service mode in ten different nights. Three of the observing nights (16 Dec. 2003, 5 Nov. 2003, 19 Feb. 2004; MJD = 52928, 52948, 53 054) were categorized as dry (relative humidity below 10\%), and five (25 Dec. 2003, 12 Jan. 2004, 26 Feb. 2004, 7 Mar. 2004, 12 Mar. 2004; MJD = 52 998,
Table 2. Observations: NACO.

\begin{tabular}{lrccr}
\hline \hline & Date & Filter, SR & Set-up & $\begin{array}{r}\text { Exposure- } \\
\text { time }\end{array}$ \\
\hline HD 30505 & 7 Mar. 2004 & $J, 12.2 \%$ & coro & $800 \mathrm{~s}$ \\
HIP 16908 & 5 Nov. 2003 & $J, 17.9 \%$ & direct & $1586 \mathrm{~s}$ \\
HD 286589 & 25 Dec. 2003 & $J, 11.7 \%$ & direct & $1586 \mathrm{~s}$ \\
LP 415-176 & 5 Nov. 2003 & $J, 11.5 \%$ & direct & $2061 \mathrm{~s}$ \\
HIP 16548 & 16 Dec. 2003 & $J, 12.0 \%$ & coro & $960 \mathrm{~s}$ \\
V1102 Tau & 12 Mar. 2004 & $J, 11.9 \%$ & coro & $960 \mathrm{~s}$ \\
HAN 513 & 26 Feb. 2004 & $J, 11.6 \%$ & direct & $1586 \mathrm{~s}$ \\
HAN 172 & 10 Feb. 2004 & $J, 11.9 \%$ & coro & $960 \mathrm{~s}$ \\
HAN 513 & 26 Feb. 2004 & $J, 11.5 \%$ & coro & $960 \mathrm{~s}$ \\
HIP 15720 & 5 Nov. 2003 & $J, 17.9 \%$ & coro & $960 \mathrm{~s}$ \\
BD+08 642 & 5 Nov. 2003 & $J, 12.0 \%$ & coro & $960 \mathrm{~s}$ \\
BD+16 630 & 19 Feb. 2004 & $J, 11.7 \%$ & coro & $960 \mathrm{~s}$ \\
J 332 & 9 Feb. 2004 & $J, 12.0 \%$ & coro & $960 \mathrm{~s}$ \\
BD+16 630 & 19 Feb. 2004 & $J, 11.6 \%$ & coro & $960 \mathrm{~s}$ \\
LP 415-378 & 12 Jan. 2004 & $J, 11.7 \%$ & direct & $1586 \mathrm{~s}$ \\
\hline
\end{tabular}

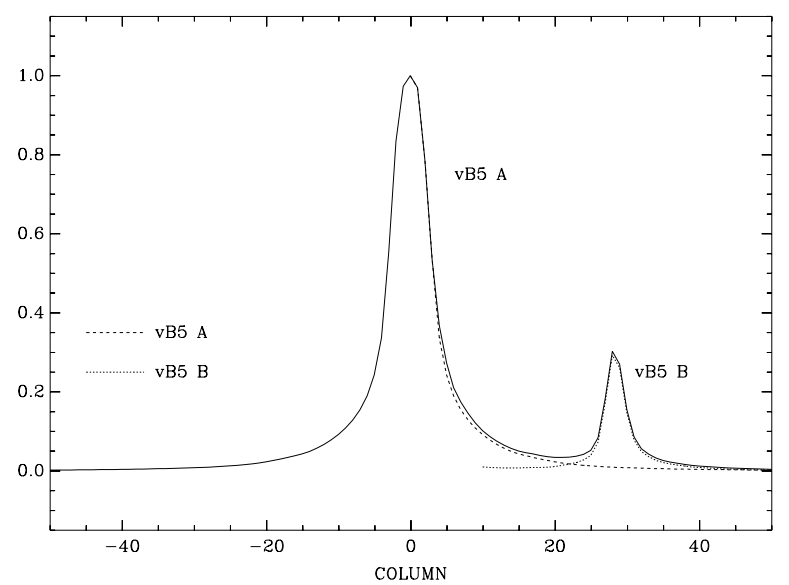

Fig. 1. The figure shows how the fluxes of the primary and secondary are measured individually. The full-line shows a cut through the image of HIP16908 A. The two dashed lines are: 1) the profile of the A-component after subtracting the B-component 2) the B-component after subtracting the A-component.

$53016,53061,53071,53076)$ as normal (relative humidity 10 to 25\%). Unfortunately J332 and HAN 172 (9 Feb. 2004, 10 Feb. 2004; MJD = 53 044, 53 045) were observed during wet conditions (relative humidity $56 \%$, and $62 \%$ ). These two nights were also partly non-photometric. The accuracy of the photometry of these two stars is thus limited. For the other nights, we could use the fluxes of the primaries (when not saturated, and when taken without the mask), as well as observations of ESO's flux-standard stars taken at the beginning of each night, to flux-calibrate the images. In the case of stars observed with the mask, only the standard stars could be used and thus, the photometric errors are a bit higher. Figure 1 shows a typical example how the fluxes of the two components of a binary are measured individually. The errors of the separation and position angle for the binaries given in Table 4 were determined from the variance of the individual exposures and the systematic error of the determination of the image scale and image tilt given above. 
Table 3. Observations: Keck.

\begin{tabular}{lcccc}
\hline \hline Name & Date & Filter, SR & Set-up & $\begin{array}{c}\text { Exposure- } \\
\text { time }\end{array}$ \\
\hline HD 28099 & 8 Dec. 2003 & H2 $(1-0), 37 \%$ & direct & $80 \mathrm{~s}$ \\
HD 30505 & 8 Dec. 2003 & $K^{\prime}, 41 \%$ & direct & $64 \mathrm{~s}$ \\
HD 286589 & 8 Dec. 2003 & H2 $(1-0), 27 \%$, & direct & $80 \mathrm{~s}$ \\
\hline
\end{tabular}

\subsection{Direct imaging: Keck II}

In addition to the NACO AO-observations, HD 30505, HD 286589 and HD 28099 were observed with the Keck II telescope on Dec. 8, 2003. We used the narrow field (pixel scale $0.00993^{\prime \prime} \pm 0.00005^{\prime \prime}$; Ghez et al. (2004) NIRC2 facility camera ${ }^{2}$ which accepts the corrected beam from the adaptive AO-system (Wizinowich et al. 2000). The weather conditions throughout the night were marked by increasing amounts of clouds which impact the AO-system performance. The first two targets observed - HD 28099 and HD 30505 - could be observed with the AO-system wavefront sensor operating at the fastest rate, however, extinction from clouds forced the last target - HD 286589 - to be observed at a reduced update rate. The lower sampling rate resulted in a slightly broader FWHM for this source. In contrast to NACO, objects of lower mass can be detected with NIRC2 if the $K^{\prime}$ band is used. This is because the Strehl ratio improves at longer wavelength. The other reason for using the $K^{\prime}$ band is that for those objects, which were observed with both telescope, we obtain color information. Exposure times were chosen to avoid saturation on the bright primary star. The exposures where continued, even when a companion became visible in order to search for possible additional, fainter companions.

The exposure time listed in Table 3 is the sum of the eight individual exposures taken at four different array positions. For HD 28099 and HD 30505 an AO-rate of $672 \mathrm{~Hz}$ was used, for HD 286589 we used an AO-rate of $210 \mathrm{~Hz}$. The final positions, angles, and magnitude differences listed in Table 4 are determined from a shifted and combined image created from all exposures. The uncertainties of the measurement of the positional angle and the distance are derived from the systematic error of the pixel scale $\left( \pm 0\right.$. $^{\prime} 00005$; Ghez et al. 2004), and position angle $\left( \pm 0.33^{\circ}\right.$; Lu 2004) plus the standard deviation of the measurement as derived from the measurement of each individual image. Because the conditions were not photometric, the measured $K^{\prime}$ magnitude difference and the 2MASS $K$ s primary magnitude were used to estimate the secondary magnitude; no transformations were applied.

\section{Results}

\subsection{HIP 16548}

HIP 16548 is an M0V star. We found two companion candidates west of the star (Fig. 2). The separations to the primary are $2.77 \pm 0.02$ and $2.78 \pm 0.02$ arcsec, respectively. The separation between the two companion-candidates is $0.30 \pm 0.02$ arcsec (Table 4). If true companions, the separation between the

\footnotetext{
2 see alamoana.keck. Hawaii.edu/inst/nirc2 for details
}

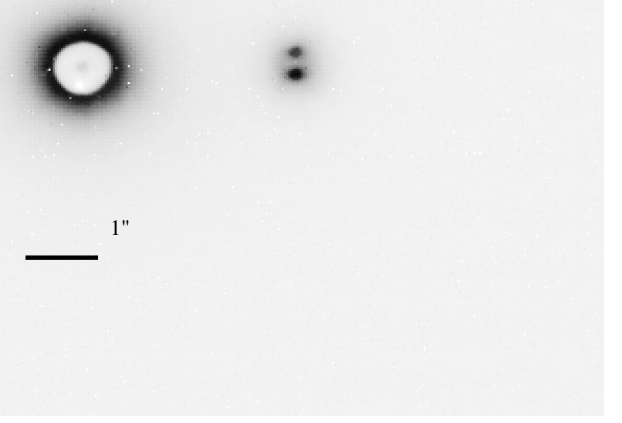

Fig. 2. The figure shows HIP 16548 A which is visible behind the semi-transparent coronographic mask together with HIP 16548 B and C. North is up and east is left in this image.

primary and the companions would be $130 \mathrm{AU}$, and the separation between the two $14 \mathrm{AU}$. The orbital periods would thus be of the order of 2000 and 100 years, respectively. The companion-candidates are $13.8 \pm 0.3$ and $14.0 \pm 0.3 \mathrm{mag}$ in the $J$-band. This would correspond to absolute magnitudes $M_{\mathrm{J}}$ of 10.5 and $10.7 \mathrm{mag}$, which implies masses below $0.1 M_{\odot}$.

We took a spectrum with the MIKE (Magellan Inamori Kyocera Echelle) spectrograph on the Magellan $6.5 \mathrm{~m}$ telescope. The resolution is $\lambda / \Delta \lambda$ is 30000 at $7000 \AA$ with a slit width of 0.7 arcsec. Thus, both components were in the slit. The integration time was one hour, which gave a $S / N$ of about 20. Following Reid et al. (1995) and Hawley et al. (1996), the spectral types of $\mathrm{M}$ stars can be determined by measuring the height of the TiO-bandheads. The $\mathrm{TiO} 2$ and TiO5-coefficients are the ratios of the fluxes TiO2 $=F[7043-7046 \AA] / F[7058-7061 \AA]$ and TiO5 $=F[7042-7046 \AA] / F[7126-7135 \AA]$. Figures 3 and 4 show the $\mathrm{TiO} 2$ and $\mathrm{TiO} 5$-coefficients versus the spectral type. As can be seen in the figures, both coefficients decrease up to a spectral-type of M 7V and then increase again. For a given coefficient, there could be two possible spectral types, and hence masses. Reid et al. (1995) and Hawley et al. (1996) both limit their analysis to stars earlier than M 7V. We use our own library of spectra (Guenther \& Wuchterl 2003) to expand the method up to spectral type L. The dashed lines in Figs. 3 and 4 give the $\mathrm{TiO} 2$ and $\mathrm{TiO} 5$-values obtained for HIP $16548 \mathrm{~B}$ and C. The TiO-coefficients give as possible solutions a spectraltype $\mathrm{M} 9 \mathrm{~V}$ to $\mathrm{LOV}$, and $\mathrm{M} 4 \mathrm{~V}$ to $\mathrm{M} 5 \mathrm{~V}$. In order to find out which of two possibilities is the correct one, we additionally used the PC3-coefficients from Martín et al. (1996). From the PC3-coefficients $(\mathrm{PC} 3=F[8230-8270 \AA] / F[7540-7580 \AA]$; $\left.\mathrm{SpT}=-6.685+11.715 \times(\mathrm{PC} 3)-2.024 \times(\mathrm{PC} 3)^{2}\right)$, we obtained a spectral type $\mathrm{M} 8 \mathrm{~V}$ to $\mathrm{M} 9 \mathrm{~V}$.

The fact that we now know that the objects have a spectral type of M $8 \mathrm{~V}$ to $\mathrm{M} 9 \mathrm{~V}$ allows us to test the quality of the photometry. According to Dahn et al. 2002 an objects with a spectral type in the range between $\mathrm{M} 8 \mathrm{~V}$ and $\mathrm{M} 9 \mathrm{~V}$ have an $M_{\mathrm{J}}$ 
Table 4. Results.

\begin{tabular}{|c|c|c|c|c|c|c|c|c|c|}
\hline & Type & $\begin{array}{c}\text { Prim }^{5} \\
\text { spec }\end{array}$ & $\begin{array}{c}\text { Prim }^{5} \\
m_{J} \\
\end{array}$ & $\begin{array}{c}\text { Prim }^{5} \\
m_{K^{\prime}} \\
\end{array}$ & $\mathrm{PA}$ & $\begin{array}{c}\text { Separation } \\
\text { [arcsec] }\end{array}$ & $\begin{array}{c}\text { Secon. }^{6} \\
m_{J} \\
\end{array}$ & $\begin{array}{c}\text { Secon. } \\
m_{K^{\prime}} \\
\end{array}$ & $\begin{array}{c}\text { Secon. } \\
\text { spec }\end{array}$ \\
\hline HD 28099 & binary & G2V & & 6.6 & $191.5 \pm 0.5^{\circ}$ & $0.43 \pm 0.01$ & & $11.47 \pm 0.09$ & M 7V-M 8V \\
\hline HD 30505 & binary & & 8.2 & 7.1 & $107.2 \pm 0.4^{\circ}$ & $0.408 \pm 0.005$ & & $11.97 \pm 0.10$ & M 7V \\
\hline HIP 16908 & binary & $\mathrm{K} 2 \mathrm{~V}$ & 7.8 & & $266.2 \pm 1.0^{\circ}$ & $0.77 \pm 0.02$ & $10.4 \pm 0.1$ & & M 1V-M 3V \\
\hline HD 286589 & binary & $\mathrm{K} 5 \mathrm{~V}$ & 8.7 & & $247.8 \pm 0.5^{\circ}$ & $0.62 \pm 0.02$ & $12.1 \pm 0.1$ & & M 5V-M 6V \\
\hline & & & & 7.7 & $248.5 \pm 0.5^{\circ}$ & $0.63 \pm 0.07$ & & $10.48 \pm 0.10$ & M 5V-M 6V \\
\hline LP 415-176 & binary & $\mathrm{K} 2 \mathrm{~V}$ & 10.0 & & $322.4 \pm 0.1^{\circ}$ & $4.26 \pm 0.03$ & $11.9 \pm 0.1$ & & M 7V-M 8V \\
\hline HIP 16548 & triple & M OV & 9.2 & & $268.1 \pm 0.1^{\circ}$ & $2.77 \pm 0.02$ & $13.8 \pm 0.3$ & & M 8V-M 9V \\
\hline & & & & & $274.3 \pm 0.1^{\circ}$ & $2.78 \pm 0.02$ & $14.0 \pm 0.3$ & & M 8V-M 9V \\
\hline V1102 Tau & binary & M $1 \mathrm{~V}$ & 9.2 & & $78.6 \pm 0.5^{\circ}$ & $1.60 \pm 0.03$ & $10.5 \pm 0.3$ & & $\mathrm{M} 2 \mathrm{~V}-\mathrm{M} 4 \mathrm{~V}$ \\
\hline HAN 513 & binary & $\mathrm{M} 0.5 \mathrm{~V}$ & 9.3 & & $25.9 \pm 1.3^{\circ}$ & $0.52 \pm 0.02$ & $11.0 \pm 0.1$ & & M 2V-M 4V \\
\hline HAN 172 & binary $^{4}$ & M 1V & 9.5 & & $106.0 \pm 0.8^{\circ}$ & $0.83 \pm 0.02$ & $13.4 \pm 0.5$ & & M 5V-M 9V \\
\hline HIP 15720 & binary? ${ }^{1}$ & K7V & 6.3 & & & & & & \\
\hline $\mathrm{BD}+08642$ & binary $^{2}$ & K5V & 8.0 & & & & & & \\
\hline $\mathrm{BD}+16630$ & binary $?^{3}$ & & 10.0 & & & & & & \\
\hline J 332 & active $^{4}$ & K5V & 9.4 & & & & & & \\
\hline LP 415-378 & active & MOV & 9.6 & & & & & & \\
\hline
\end{tabular}

${ }^{1}$ The stellar image is a little elongated. The stellar image is $0.120 \times 0.140 \mathrm{arcsec}$, the elongation has a PA of $210 \pm 10^{\circ}$.

${ }^{2}$ The stellar image is a little elongated. The stellar image is $0.113 \times 0.157 \mathrm{arcsec}$, the elongation has a PA of $165 \pm 10^{\circ}$.

${ }^{3}$ This star shows a relatively large amplitude of the RV-variation and thus could be a short period binary.

${ }^{4}$ This image was taken under bad conditions, limiting the accuracy of the photometry.

${ }^{5}$ Value for primary. ${ }^{6}$ Value for secondary.

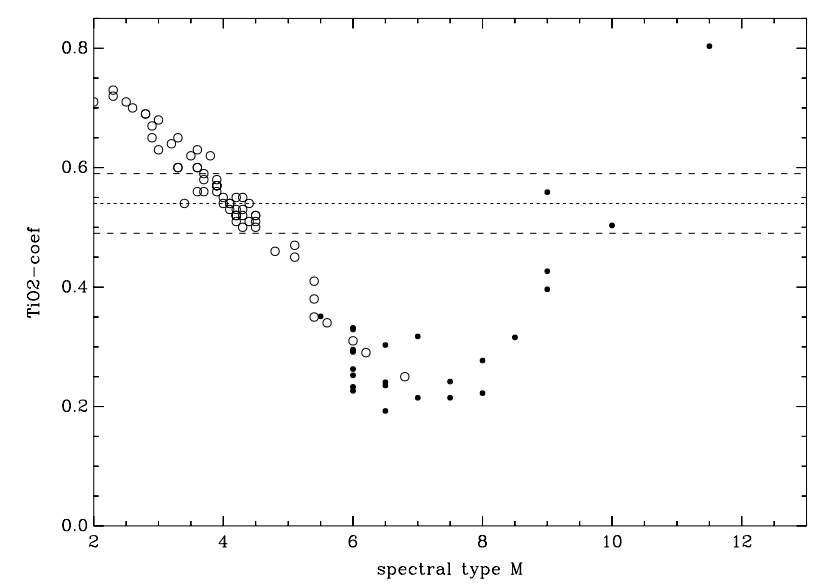

Fig. 3. The dashed line shows the $\mathrm{TiO} 2$-coefficient together with the errors. The circles are values obtained for $\mathrm{M}$ stars from Reid et al. (1995) and Hawley et al. (1996) for Hyades stars, and the filled dots for very low-mass stars from Guenther \& Wuchterl (2003). The spectral type thus is between M9 V and LOV, in good agreement with the brightness of the object in the $J$-band.

in the range between 10.5 and $11.8 \mathrm{mag}$. For HIP $16548 \mathrm{~A}$ and HIP $16548 \mathrm{~B}$ we derive $M_{\mathrm{J}}=10.5 \pm 0.3$ and $M_{\mathrm{J}}=10.8 \pm 0.3$ using the distance of the Hyades and the values given in Table 4. The agreement between the spectroscopic and photometric determination of the spectral type thus is excellent. The objects have masses between 0.07 to $0.08 M_{\odot}$ (Chabrier et al. 2000), which puts the objects very close to the brown dwarf boarder. We do not, however, see the Li I 6708 line, so it is unclear as to whether the objects are indeed brown dwarfs (Fig. 6). While the presence of $\mathrm{Li}$ is most common in brown dwarfs, a few older late-M and $\mathrm{L}$ dwarfs have been detected which do not show $\mathrm{Li}$,

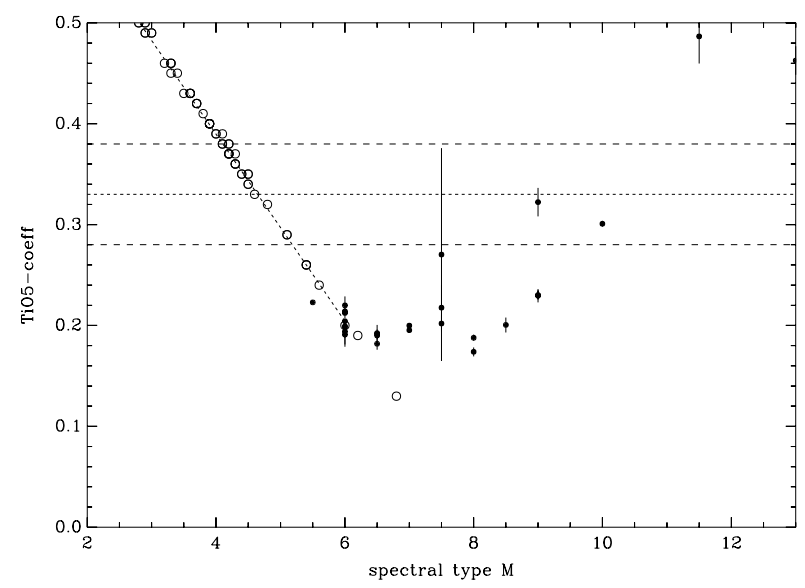

Fig. 4. Same as Fig. 3 but for the TiO5-coefficient. We find again a possible solution for the spectral type of $\mathrm{M} 9 \mathrm{~V}$ and $\mathrm{LOV}$.

including DENIS-P J1208+0149 (M 9) and DENIS-P J00900658 (L0), Martín et al. (1999).

The spectra of HIP $16548 \mathrm{~B}$ and $\mathrm{C}$ shows $H \alpha$ in emission. The equivalent width is $-2.3 \AA$, which is fairly typical for such late-type objects (Fig. 5). The strongest atomic lines are the NaI doublet at 8183 and $8195 \AA$ (Fig. 7) and the KI resonance doublet at 7665 and $7699 \AA$ (Fig. 8), which are not only clearly visible in the spectrum of HIP 16548 B,C but also have roughly the same equivalent width as of LP944-20. Another characteristic feature of young brown dwarfs is the CsI 8521 $\AA$-line (Fig. 9). This line is however not seen in the spectrum of HIP 16548 B,C. 


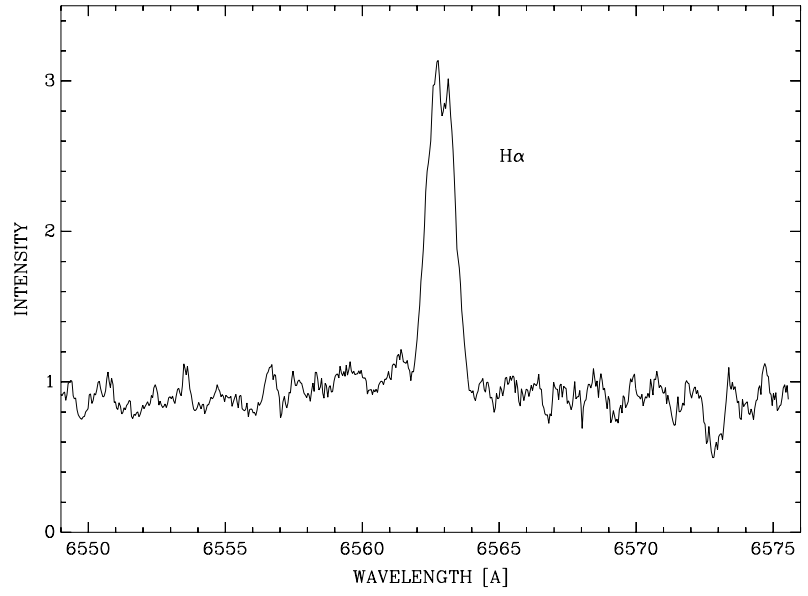

Fig. 5. The figure shows the $\mathrm{H} \alpha$-emission line of HIP 16548 B,C.

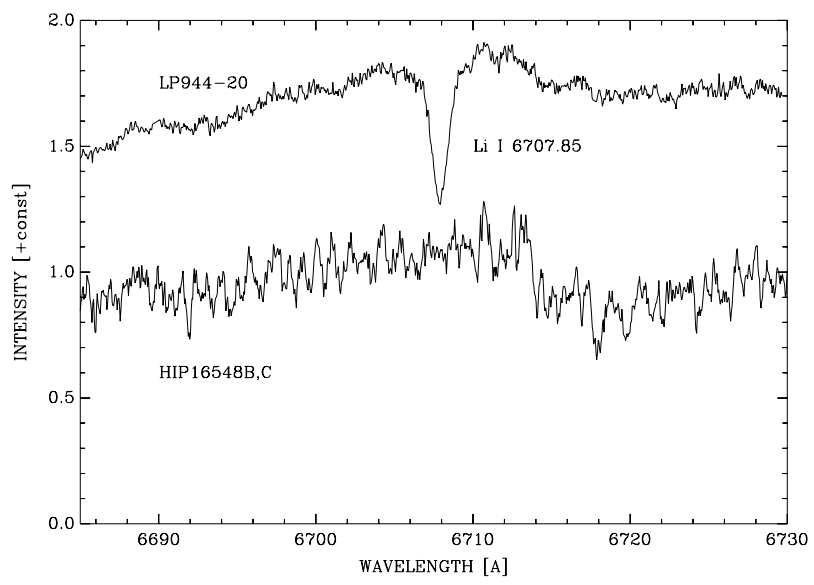

Fig. 6. The figure shows the Li I $6708 \AA$ line in the spectrum of the brown dwarf LP944-20 which has about the same age and the same spectral type as HIP $16548 \mathrm{~B}, \mathrm{C}$. This line is not seen in the spectrum of HIP $16548 \mathrm{~B}, \mathrm{C}$, which is shown below.

\subsection{The other companion candidates}

Table 4 gives an overview of the values obtained for the stars. HD 28099 is a G2V star. The companion has a $K^{\prime}$ magnitude of $11.97 \pm 0.10$ which implies that it is a $\mathrm{M} 7 \mathrm{~V}$ to $\mathrm{M} 8 \mathrm{~V}$ star.

HD 30505 was observed with both instruments. Because the separation is $0.408 \pm 0.005$ arcsec the secondary was behind the coronographic mask in the NACO observations. Although NACO image is slightly elongated, we prefer to use the Keck data only, which were taken without the mask. The companion has $K^{\prime}$ magnitude of $11.47 \pm 0.09$, which implies that the companion is an $\mathrm{M} 7 \mathrm{~V}$ star.

HIP 16908 is a $\mathrm{K} 2 \mathrm{~V}$ star, and its companion has an $M_{\mathrm{J}}=$ $7.1 \pm 0.3$, and thus a M $1 \mathrm{~V}$ to $\mathrm{M} 3 \mathrm{~V}$-star. This binary was first detected by Patience et al. (1998) in a speckle interferometry survey of the Hyades. These authors estimate the companion equal to $0.3 M_{\odot}$, which is in good agreement with our measurement. No other companion was found. We searched for a possible 3rd companion, but none was found in our observations.

HD 286589 is another star which we observed with both instruments. Figure 10 shows the $J$-band image and Fig. 11 the

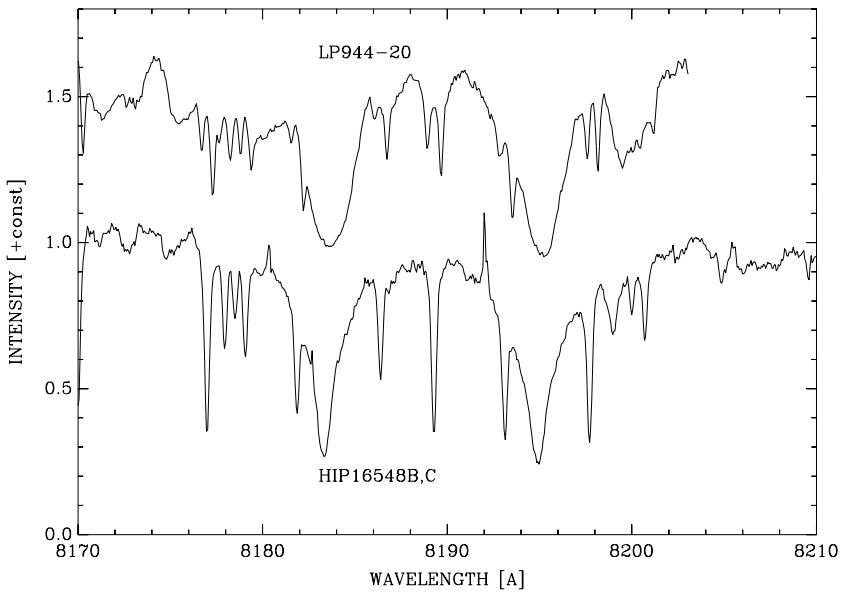

Fig. 7. The figure shows the NaI doublet at 8183 and $8195 \AA$ and for comparison in the spectrum of the brown dwarf LP944-20 is shown below.

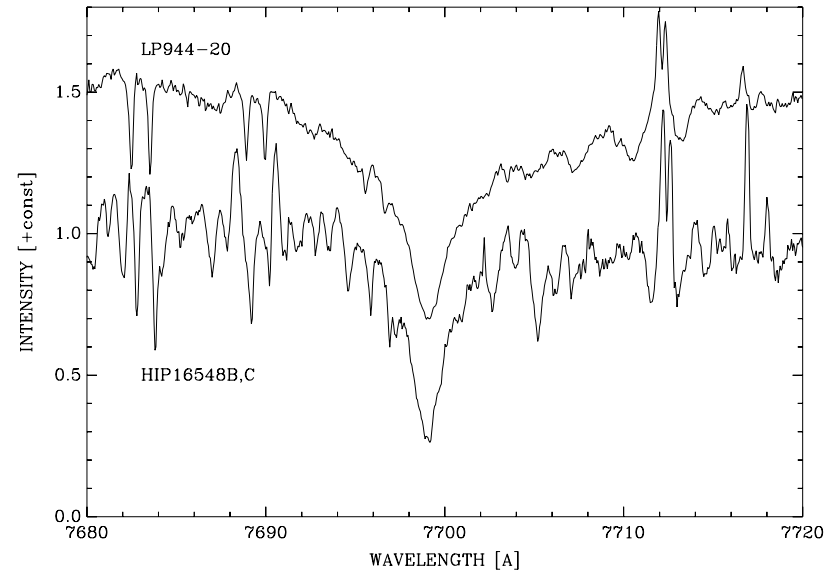

Fig. 8. The figure shows the KI 7699 line in the spectrum of HIP 16548 B,C and for comparison in the spectrum of the brown dwarf LP944-20 which has about the same age and the same spectral type as HIP 16548 B,C.

$K^{\prime}$-band image. North is up and East is left in both images. From the images we obtain position angles of $247.8 \pm 0.5^{\circ}$ and $248.5 \pm 0.4^{\circ}$, and separations of $0.62 \pm 0.02$ and $0.63 \pm 0.07$ arcsec, respectively. The agreement between the two data-sets is thus excellent. We determine a $J$-band magnitude of $12.1 \pm 0.1$ mag, and a $K^{\prime}$-band magnitude of $10.48 \pm 0.10 \mathrm{mag}$. We thus conclude that the K5V star HD 286589 is orbited by a M 5V to M6V star. The projected separation currently is $29 \mathrm{AU}$.

The K2V star LP 415-176 is also a binary. The separation is $4.26 \pm 0.03 \mathrm{arcsec}$. The $J$-band magnitude of the companion is $11.9 \pm 0.1 \mathrm{mag}$ which implies that it is a $\mathrm{M} 7 \mathrm{~V}$ to $\mathrm{M} 8 \mathrm{~V}$ star. Our aim was to searched for additional components but did not find any. V1102 Tau is an M $1 \mathrm{~V}$ star. In this case, we found a companion-candidate with $M_{\mathrm{J}}=7.2 \pm 0.3 \mathrm{mag}$, which corresponds to an M $2 \mathrm{~V}$ to M $4 \mathrm{~V}$ star. HAN 513 is an M $0.5 \mathrm{~V}$ star with a M 2 to $\mathrm{M} 4 \mathrm{~V}$ companion.

HAN 172 is an M 1V star, which was observed in less than ideal conditions. Nevertheless, we found one stellar companion-candidate with an absolute $J$-magnitude of $10.1 \pm$ $0.5 \mathrm{mag}$. The companion is presumably an $\mathrm{M} 5 \mathrm{~V}-\mathrm{M} 9 \mathrm{~V}$ star, 


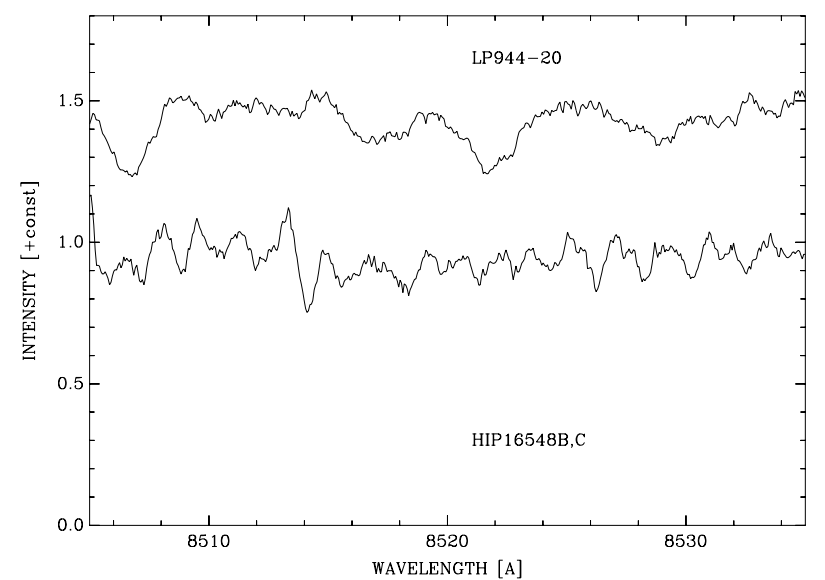

Fig. 9. The figure shows the region around the CsI $8521 \AA$-line for HIP 16548 B,C and LP944-20. This line is not seen in the spectrum of HIP 16548 B,C.

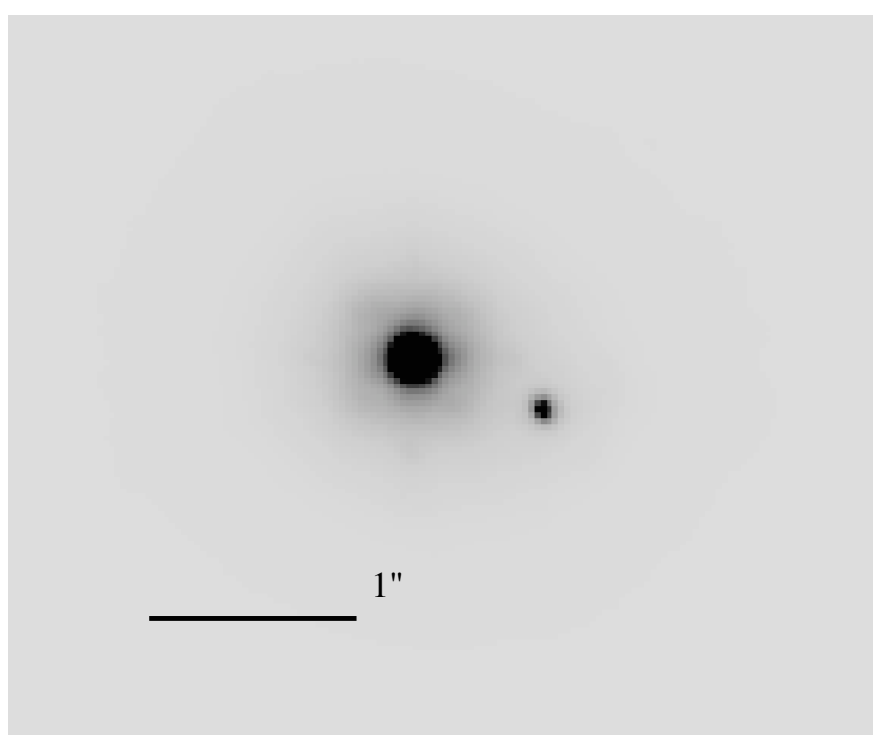

Fig. 10. $J$-band image of HD 286589. A $K^{\prime}$-band image of this object is shown in Fig. 11. North is up and East is left. The primary is a K5V star, the companion a M $5 \mathrm{~V}$ to M $6 \mathrm{~V}$ star, the separation is $0.62 \pm 0.02$ arcsec, which corresponds to a projected distance 29 AU. The size of the image is $3.8 \times 3.8$ arcsec.

and has a mass between 0.08 to $0.12 M_{\odot}$ (Chabrier et al. 2000). Unfortunately the star was observed with the mask when the conditions were less than ideal so that the accuracy of the photometry is rather limited. The mass of the object is close to the brown dwarf boarder but the object probably is not a brown dwarf. Usually, an object with a mass higher than $0.075 M_{\odot}$ is considered a star, and an object with a mass lower than $0.070 M_{\odot}$ a brown dwarf but the exact position of the boundary depends on metallicity (Chabrier et al. 2000). This means that at the age of the Hyades, the boundary is somewhere around M 8V, or M 9V. The brown dwarf LP944-20 which has about the same age as the Hyades has a spectral type of M $9 \mathrm{~V}$. The mass of it is between 0.056 and $0.064 M_{\odot}$ (Tinney \& Reid 1998). DENIS-P J144137.3-094559 which recently turned out to be member of the super Hyades has a mass of $0.072_{-0.022}^{+0.026} M_{\odot}$ and a spectral type of L1V (Seifahrt et al. 2005).

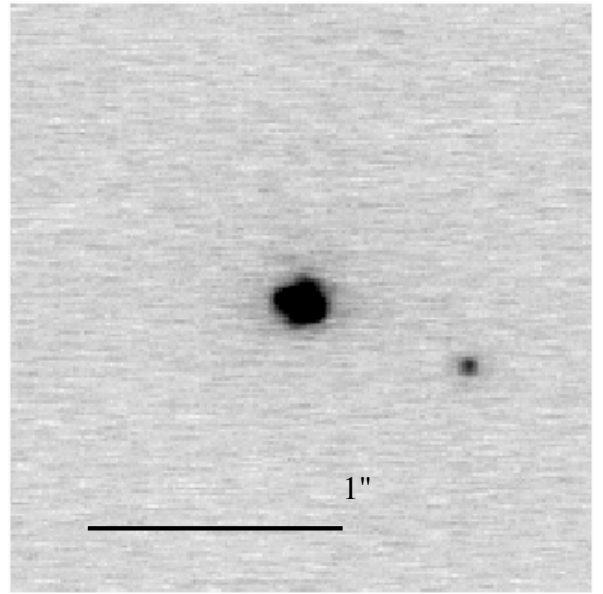

Fig. 11. This is the $K^{\prime}$-band image of HD286589, which can be compared with the $J$-band image shown in Fig. 10. North is up and East is left. The separation is $0.63 \pm 0.07$ arcsec. The size of the image is $3.5 \times 3.5$ arcsec.

\subsection{Objects without companion candidates}

Figure 12 shows the RV measurements for those stars, where we did not find obvious companion candidates (BD+16630, LP 415-378, HIP 15720, and BD+08 642). $\mathrm{BD}+16630$, HIP 15720, and BD+08 642 were however observed with the semi-transparent coronographic mask so that we can not fully exclude the presence of a faint companion with a separation of less than 0.35 arcsec.

The stellar images of HIP 15720 and BD+08 642 are slightly elongated. Because the coronographic mask is semitransparent, the images of the stars are visible, and not saturated. In the case of HIP 15720 the image is $17 \%$ longer in one direction than in the other, and in the case of BD+08642 the difference is $40 \%$. These stars thus might also be binaries. While the elongation of the images is larger than the typical $\leq 10 \%$ elongation of the other stellar images, only further observations will show whether also these objects are binaries or not.

No indications for binarity were found in the image for J332, LP 415-378, and BD+16 630. As mentioned above, J332 was observed in less than ideal conditions. The $R V$-variations of J332 and LP 415-378 are most likely caused by stellar activity. The low level of the variations in J332 are consistent with previous results (Paulson et al. 2002). Figure 13 shows the S-index (Paulson et al. 2002) versus the RV for LP 415-378. There is a clear trend of activity with RV which indicates that the large amplitude in RV for this star is most likely caused by stellar activity.

In the case of BD+16630 we have only three RV measurements. However, the relatively large amplitude implies that this object probably is a short-period binary, and the separation of two components is simply to small.

\section{Conclusions}

The aim of the project is to search for brown dwarf companions orbiting Hyades stars within $8 \mathrm{AU}$ using precise $R V$ 


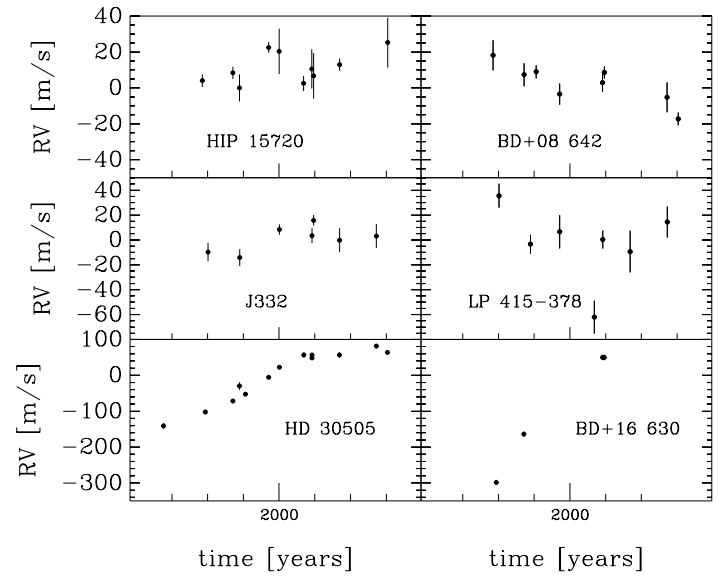

Fig. 12. This figure shows the RV measurements obtained for those stars, were we did not find obvious companion candidates in the direct images, and HD 30505 for comparison. In the case of BD+08 642 and HIP 15720 the stellar images are slightly elongated, which may imply that they are binaries. The fact that the RV-amplitude of the three datapoints of $\mathrm{BD}+16630$ are large implies that this object probably is a short-period binary. In the case of J332 and LP 415-378, we argue that the RV-variations are caused by stellar activity.

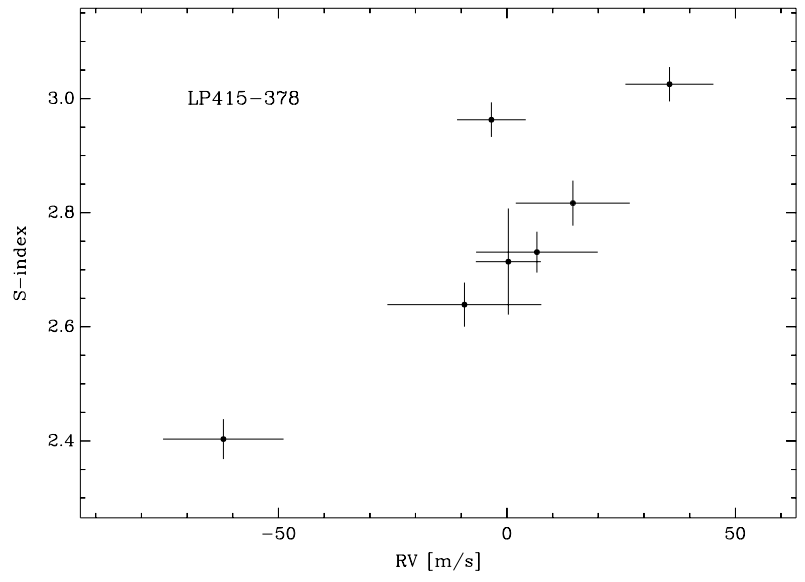

Fig. 13. The figure shows the S-index versus the RV for LP 415-378. The correlation between the two indicates that the RV-variations are probably caused by stellar activity.

measurements as input information. If the distance would be $\leq 3$ AU we would have observed a full orbit, and semi-amplitude of the $R V$-variations would be larger than $200 \mathrm{~m} / \mathrm{s}$. Such an object would be easily detectable in the $R V$ data, and we can rule out such an object for all 98 Hyades stars observed. Brown dwarfs orbiting at a distance between $3 \mathrm{AU}$ and $8 \mathrm{AU}$, would show up as trends of the $R V$. The problem, however is that such trends of the $R V$ can also be caused by stellar companions. The idea of the project is search for the stellar companions which can easily be detected by means of AO-imaging, because they are relatively bright, and have a separation $\geq 0.5$ arcsec. The detection of a stellar companion thus implies that $R V$ trend is caused by this companion and not by a brown dwarf. An object with a low-amplitude $R V$ trend, without a stellar companion would then be a brown dwarf companion candidate.
From an RV survey of 98 stars, we have selected all the stars that could possibly have a long-term trends in RV, 14 stars in total. In nine of these we found companions. One of these stars has two companions with masses between 0.07 to 0.08 $M_{\odot}$. These objects are thus close to the boarder to the brown dwarf regime, though without the presence of $\mathrm{Li}$ and $\mathrm{Cs}$, we can not confirm that they are indeed brown dwarfs. Another star is orbited by a companion with a mass between 0.08 to $0.10 M_{\odot}$. In this respect it is interesting to note that the $\mathrm{dM} 4.5 \mathrm{e}$ star G124-62 which is orbited by two L-dwarfs of $0.072_{-0.022}^{+0.026}$ $M_{\odot}$ recently turned out to be a member of the super Hyades (Seifahrt et al. 2005). From the remaining five stars, two show evidence that the RV-variations are caused by stellar activity, and one star seems to be a short-period binary. Thus there are only two stars, which might have sub-stellar companions. However, the NACO images of these stars are elongated which might imply that also these could be binaries.

From the RV-data alone, we can exclude that any of the 98 Hyades stars studied has a brown dwarf companion with distance of $\leq 3 \mathrm{AU}$. From the 14 stars selected, at least 9 and possibly 12 have companions with masses $\geq 0.07 M \odot$, and the two remaining ones are just active. We did find three objects which are close to the boarder between stars and brown dwarfs. Assuming that the stars with elongated images are also binaries, we can also exclude brown dwarf companions orbiting at a distance between 3 and $8 \mathrm{AU}$ for all stars observed. We thus estimate the number of companions with masses between $10 M_{\mathrm{J}}$ and $70 M_{\mathrm{J}}$ with separations between $\leq 8 \mathrm{AU}$ in the Hyades as $\leq 2 \%$. We found three companions with masses between 0.08 to $0.10 M_{\odot}$ at separations of 130 and $40 \mathrm{AU}$. The frequency of such objects in the Hyades thus is about $3 \pm 2 \%$.

As mentioned above there is a general agreement that the frequency of brown dwarfs orbiting old stars in the solar neighbourhood at distances of $\leq 3 \mathrm{AU}$ is about $\leq 0.5 \%$. In here we have first of all show that there is also a brown dwarf desert for stars in the Hyades which are much younger and have a higher metallicity than the stars in the solar neighbourhood, and secondly we show that the brown dwarf desert extends outwards at least up to $8 \mathrm{AU}$. Whether the brown dwarf desert extends even further outwards can not be said on the basis of this survey.

Acknowledgements. We are grateful to the user support group of the VLT. Some of the data presented in this paper were obtained at the W. M. Keck Observatory, which is operated as a scientific partnership among the California Institute of Technology, the University of California, and the National Aeronautics and Space Administration. The Observatory was made possible by the generous financial support of the W. M. Keck Foundation. This work made use of the SIMBAD database operated by CDS, France, and data products from the Two Micron All Sky Survey, which is a joint project of the University of Massachusetts and the Infrared Processing and Analysis Center/California Institute of Technology, funded by the National Aeronautics and Space Administration and the National Science Foundation. Funding for J.P.'s fellowship was provided to the Michelson Science Center under its Michelson Fellowship Program. Portions of this material is based upon work supported by the National Aeronautics and Space Administration under Grant NNG04G141G issued through the Terrestrial Planet Finder Foundation Science program, as well as under the auspices of the U.S. Department of Energy by the University of California, Lawrence Livermore National 
Laboratory under Contract W-7405-ENG-48, and also supported in part by the National Science Foundation Science and Technology Center for Adaptive Optics, managed by the University of California at Santa Cruz under cooperative agreement No. AST 9876783.

\section{References}

Abt, H. A., \& Levy, S.G. 1976, ApJS, 30, 273,

Boesgaard, A. M., \& Budge, K. G. 1988, ApJ, 332, 410

Burrows, A., Marley, M., Hubbard, W. B., et al. 1997, ApJ, 491, 856

Cayrel, R., Cayrel de Strobel, G., \& Campbell, B. 1985, A\&A, 146, 249

Chabrier, G., Baraffe, I., Allard, F., \& Hauschildt, P. 2000, ApJ, 542, 464

Chauvin, G., Lagrange, A.-M., Dumas, C., et al. 2005a, A\&A, 438, L25

Chauvin, G., Lagrange, A.-M., Zuckerman, B., et al. 2005b, A\&A, 438, L29

Claudi, R. U., Costa, J., Feldt, M., et al. 2004, in Second Eddington Workshop: Stellar structure and habitable planet finding, ed. F. Favata, S. Aigrain and A. Wilson (Noordwijk: ESA Publications Division), ESA SP-538, 301

Close, L., Lenzen, R., Guirado, J. C., et al. 2005, Nature, 433, 286

Cochran, W. D., Hatzes, A. P., \& Paulson, D. B. 2002, AJ, 124, 565

Dahn, C. C., Harris, H. C., Vrba, F. J., et al. 2002, AJ, 124, 1170

Duquennoy, A., \& Mayor, M. 1991, A\&A, 248, 485

Endl, M., Hatzes, A. P., Cochran, W., et al. 2004, ApJ, 611, 112

Fischer, D. A., \& Marcy, G. W. 1992, ApJ, 396, 178

Freed, M., Close, L. M., \& Siegler, N. 2003, ApJ, 584, 453

Ghez, A. M., Wright, S. A., Matthews, K., et al. 2004, ApJ, 601, L159

Goto, M., Kobayashi, N., Terada, H., et al. 2002, ApJ, 567, L59

Guenther, E. W., Neuhäuser, R., Huélamo, N., Brandner, W., \& Alves, J., 2001, A\&A, 365, 514

Guenther, E., \& Wuchterl, G., 2003, A\&A, 401, 677

Hawley, S. L., Gizis, John E., \& Reid, I. Neill 1996, AJ, 112, 2799

Henry, T. J., \& McCarthy, D. W. 1993, AJ, 106, 773.

Kirkpatrick, J. D., Dahn, C. C., Monet, D. G., et al. 2001, AJ, 121, 3235

Lineweaver, Ch., \& Grether, D. 2003, ApJ, 598, 1350

Lebreton, Y., Fernandes, J., \& Lejeune, T. 2001, A\&A, 374, 540

Liu, M. C., Fischer, D. A., Graham, J. R., et al. 2002, ApJ, 571, 519

Lowrance, P. J., McCarthy, C., \& Becklin, E. E. 1999, ApJ, 512, L69

Lowrance, P. J., Schneider, G., Kirkpatrick, J. D., et al. 2000, ApJ, 541, L390

Lu, J. 2004, personal communication

Masciadri, E., Mundt, R., Henning, Th., et al. 2005, ApJ, 625, 1004
McCarthy, C., \& Zuckerman, B. 2004, AJ, 127, 2871

McCaughrean, M. J., Close, L. M., Scholz, R.-D., et al. 2004, A\&A, 413, 1029

Malkov, O., Piskunov, A., \& Zinnecker, H. 1998, A\&A, 338, 452

Marcy, G., Butler, R. P., Fischer, D. A., \& Vogt, S. S. 2003, in ASP Conf. Ser., Scientific Frontiers in Research on Extrasolar Planets, ed. D. Deming, \& S. Seager (San Francisco: ASP)

Martín, E. L., Rebolo, R., \& Zapatero-Osorio, M. R. 1996, ApJ, 469, 706

Martín, E. L., Delfosse, X., Basri, G., et al. 1999, AJ, 118, 2466

Masciadri, E., Brandner, W., Bouy, H., et al. 2003, A\&A, 411, 157

Mazeh, T., Goldberg, D., Duquennoy, A., \& Mayor, M. 1992, ApJ, 401,265

Nakajima, T., Oppenheimer, B. R., Kulkarni, S. R., et al. 1995, Nature, 378,463

Neuhäuser, R., \& Guenther, E. W. 2004, A\&A, 420, 647

Neuhäuser, R., Guenther, E. W., Petr, M. G., et al. 2000, A\&A, 360, L39

Neuhäuser, R., Guenther, E. W., Alves, J., et al. 2003, Astron. Nachr. 324,535

Neuhäuser, R., Guenther, E. W., Wuchterl, G., Mugrauer, M., \& Bedalov, A. 2004, A\&A, 435, L13

Patience, J., Ghez, A. M., Reid, I. N., Weinberger, A. J., \& Matthews, K. 1998, AJ, 115, 1972

Paulson, D. B., Saar, S. H., Cochran, W. D., \& Hatzes, A. P. 2002, AJ, 124,572

Paulson, D. B., Sneden, Ch., \& Cochran, W. D. 2003, AJ, 125, 3185

Paulson, D. B., Cochran, W. D., \& Hatzes, A. P. 2004 AJ, 127, 3579.

Perryman, M. A. C., Brown, A. G. A., Lebreton, Y., et al. 1998, A\&A, 331,81

Potter, D., Martń, E. L., Cushing, M. C., et al. 2002, ApJ, 567, L133

Reid, I. N., Hawley, S. L., \& Gizis, J. E. 1995, AJ, 110, 1838

Santos, N. C., Israelian, G., \& Mayor, M. 2004, A\&A, 415, 1153

Seifahrt, A., Guenther, E., \& Neuhäuser, R., 2005, A\&A, 440, 967

Stern, S. A. 1994, AJ, 108, 2312

Tinney, C. G., \& Reid, I. N. 1998, MNRAS, 301, 1031

Torres, G., Stefanik, R. P., \& Latham, D. W. 1997, ApJ, 474, 256

Udry, S., Mayor, M., Naef, D., et al. 2002, A\&A, 390, 267

Wizinowich, P. L., Acton, D. S., Lai, O., et al. P. J. Proc. SPIE, 4007, 64

Wuchterl, G., \& Tscharnuter, W. M. 2003, A\&A, 398, 1081

Zapatero Osorio, M. R., Lane, B. F., Pavlenko, Ya., et al. 2004, ApJ, 615,958

Zucker, Sh., \&Mazeh, T. 2000, ApJ, 531, L67

Zucker, Sh., \& Mazeh, T. 2001, ApJ, 562, 1038 